\title{
Frobenius pseudo-varieties in numerical semigroups
}

\author{
Aureliano M. Robles-Pérez*† and José Carlos Rosales*ł
}

\begin{abstract}
The common behavior of several families of numerical semigroups led up to defining the Frobenius varieties. However, some interesting families were out of this definition. In order to overcome this situation, in this paper we introduce the concept of (Frobenius) pseudo-varieties. Moreover, we will show that most of the results for varieties can be generalized to pseudo-varieties.
\end{abstract}

Keywords: Pseudo-varieties, Frobenius varieties, monoids, numerical semigroups, tree associated (to a pseudo-variety).

2010 AMS Classification: 20M14.

\section{Introduction}

Let $\mathbb{N}$ be the set of nonnegative integers. A submonoid of $(\mathbb{N},+)$ is a subset of $\mathbb{N}$ that is closed for the addition and that contain the zero element. A numerical semigroup is a submonoid $S$ of $(\mathbb{N},+)$ such that $\mathbb{N} \backslash S$ is finite.

If $A$ is a non-empty subset of $\mathbb{N}$, we denote by $\langle A\rangle$ the submonoid of $(\mathbb{N},+)$ generated by $A$, that is,

$$
\langle A\rangle=\left\{\lambda_{1} a_{1}+\cdots+\lambda_{n} a_{n} \mid n \in \mathbb{N} \backslash\{0\}, a_{1}, \ldots, a_{n} \in A, \lambda_{1}, \ldots, \lambda_{n} \in \mathbb{N}\right\} .
$$

It is well known (see for instance [3, Lemma 2.1]) that $\langle A\rangle$ is a numerical semigroup if and only if $\operatorname{gcd}(A)=1$.

If $M$ is a submonoid of $(\mathbb{N},+)$ and $M=\langle A\rangle$, then we say that $A$ is a system of generators of $M$. In addition, if $M \neq\langle B\rangle$ for every $B \varsubsetneqq A$, then we say that $A$ is a minimal system of generators of $M$. It is known (see [3, Corollary 2.8]) that every submonoid of $(\mathbb{N},+$ ) has a unique minimal system of generators and, moreover, that such a system is finite.

If $S$ is a numerical semigroup, then we have that $\mathbb{N} \backslash S$ is finite. This allows us to define two notable invariants of $S$. The first one is the greatest integer that does not belong to $S$, which it is called the Frobenius number of $S$ and denoted by $\mathrm{F}(S)$. The second one is the cardinality of $\mathbb{N} \backslash S$, which is called the genus of $S$ and denoted by $\mathrm{g}(S)$. On the other hand, if $A=\left\{n_{1}<n_{2}<\ldots<n_{p}\right\}$ is the minimal system of generators of $S$, then the integers $n_{1}$ and $p$ are (another notable invariants of $S$ ) called the multiplicity and the embedding dimension of $S$ and they are denoted by $\mathrm{m}(S)$ and e $(S)$, respectively.

It is clear that, if $S$ is a numerical semigroup different from $\mathbb{N}$, then $S \cup\{\mathrm{F}(S)\}$ is also a numerical semigroup. On the other hand (see [3, Proposition 7.1]), $\bar{S}$ is a numerical semigroup with genus $g+1$ if and only if $\bar{S}=S \backslash\{x\}$, where $S$ is a numerical semigroup with genus $g$ and $x$ is an element of the minimal system of generators of $S$ that is greater than $\mathrm{F}(S)$. These facts (see [3, Proposition 7.4]) enable us to construct recursively all numerical semigroups with genus $g+1$ from all numerical semigroups with genus $g$.

The generalization of the previous situation to some distinguished families of numerical semigroups leads in [2] to introduce the concept of (Frobenius) variety.

A variety is a non-empty family $\mathcal{V}$ of numerical semigroups that fulfills the following conditions,

1. if $S, T \in \mathcal{V}$, then $S \cap T \in \mathcal{V}$;

2. if $S \in \mathcal{V}$ and $S \neq \mathbb{N}$, then $S \cup\{\mathrm{F}(S)\} \in \mathcal{V}$.

${ }^{*}$ Both of the authors are supported by FQM-343 (Junta de Andalucía), MTM2010-15595 (MICINN, Spain), and FEDER funds. The second author is also partially supported by Junta de Andalucía/Feder grant number FQM-5849.

†Departamento de Matemática Aplicada, Universidad de Granada, 18071-Granada, Spain.

E-mail: arobles@ugr.es

${ }^{\ddagger}$ Departamento de Álgebra, Universidad de Granada, 18071-Granada, Spain.

E-mail: jrosales@ugr.es 
It can be proved (see [3, Section 6.2]) that the family of Arf numerical semigroups, the family of saturated numerical semigroups, the family of numerical semigroups that are intersections of finitely many proportionally modular numerical semigroups, and the family of numerical semigroups defined by a strongly admissible (linear homogeneous) pattern are examples of varieties. However, there exist families of numerical semigroups that are not varieties, but with a very similar behavior. For example, the family of numerical semigroups with maximal embedding dimension and multiplicity equal to $m$ (see [4) is not a variety. By studying the behavior of this class of numerical semigroups, recently (see [1]) has been introduced the non-homogeneous patterns and, moreover, were defined the non-homogeneous Frobenius varieties of multiplicity $m$ (or $m$-varieties for short). All these considerations shape the starting point of the present paper. We will give the following generalization of the concept of variety.

A (Frobenius) pseudo-variety is a non-empty family $\mathcal{P}$ of numerical semigroups that fulfills the following conditions,

1. $\mathcal{P}$ has a maximum element $\max (\mathcal{P})$ (with respect to the inclusion order);

2. if $S, T \in \mathcal{P}$, then $S \cap T \in \mathcal{P}$;

3. if $S \in \mathcal{P}$ and $S \neq \max (\mathcal{P})$, then $S \cup\{\mathrm{F}(S)\} \in \mathcal{P}$.

From this definition, it is evident that a variety is a pseudo-variety. In this paper, our main goal is to show that most of the results for varieties, that were proved in [2, are generalized to pseudo-varieties.

Let us observe that, in [1, the maximum element of a $m$-variety is a numerical semigroup of the type $\{0, m, \rightarrow\}=\{0, m\} \cup\{z \in \mathbb{N} \mid z>m\}$ (where $m \in \mathbb{N} \backslash\{0\}$ ). Moreover, the multiplicity of all numerical semigroups, that belong to an $m$-variety, is the same (and equal to $m$ ). A similar remark holds for the family of numerical semigroups with maximal embedding dimension and multiplicity equal to $m$. In this work, we will show that this restriction can be removed.

As can be supposed, some results of this paper have similar proofs to the analogous ones for varieties. Nevertheless, we have preferred to write a self-contained paper which is organized as follows. In Section 2 we will study the relation between the varieties and the pseudo-varieties. In particular, we will see how to obtain the smallest variety containing a given pseudo-variety and how to get pseudo-varieties (which are not varieties) from a given variety.

Let $\mathcal{P}$ be a pseudo-variety. If $M$ is a submonoid of $(\mathbb{N},+)$, we will say that $M$ is a $\mathcal{P}$-monoid if it can be expressed as an intersection of elements of $\mathcal{P}$. In Section 3 we will introduce the concept of $\mathcal{P}$-monoid generated by a subset of $\max (\mathcal{P})$ and, moreover, we will show that every $\mathcal{P}$-monoid admits a unique minimal $\mathcal{P}$-system of generators.

In Section 4 we will see that the elements of a pseudo-variety $\mathcal{P}$ can be arranged in a tree (with root $\max (\mathcal{P})$ ), where the sons of a node $S$ of such a tree are $S \backslash\left\{x_{1}\right\}, \ldots, S \backslash\left\{x_{r}\right\}$, with $x_{1}, \ldots, x_{r}$ the elements of the minimal $\mathcal{P}$-system of generators of $S$ that are greater than $\mathrm{F}(S)$.

Finally, in Section 5 we will show that there exists the smallest pseudo-variety that contains a given family $\mathcal{F}$ of numerical semigroups. We will denote it by $\mathfrak{p}(\mathcal{F})$ and will say that it is the pseudo-variety generated by $\mathcal{F}$. We will show that, if $\mathcal{F}$ is finite, then $\mathfrak{p}(\mathcal{F})$ is finite. This fact will allow us to compute the minimal $\mathfrak{p}(\mathcal{F})$ system of generators of a given $\mathfrak{p}(\mathcal{F})$-monoid. Consequently, we will have an algorithm in order to compute $\mathfrak{p}(\mathcal{F})$ from $\mathcal{F}$.

\section{Varieties and pseudo-varieties}

From the definitions, it is clear that every variety is a pseudo-variety. However, there are pseudo-varieties that are not varieties. Indeed, if $S$ is a numerical semigroup different from $\mathbb{N}$, then $\{S\}$ is a pseudo-variety but not a variety. The next result is easy to prove.

Proposition 2.1. Let $\mathcal{P}$ be a pseudo-variety. Then $\mathcal{P}$ is a variety if and only if $\mathbb{N} \in \mathcal{P}$.

Let us remember that, if $S$ is a numerical semigroup such that $S \neq \mathbb{N}$, then $S \cup\{\mathrm{F}(S)\}$ is also a numerical semigroup. This allows to define recursively the following sequence of numerical semigroups,

- $S_{0}=S$,

- if $S_{i} \neq \mathbb{N}$, then $S_{i+1}=S_{i} \cup\left\{\mathrm{F}\left(S_{i}\right)\right\}$. 
Since $\mathbb{N} \backslash S$ is a finite set, we get a chain of numerical semigroups $S=S_{0} \varsubsetneqq S_{1} \varsubsetneqq \cdots \varsubsetneqq S_{n}=\mathbb{N}$. We will denote by $\mathcal{C}(S)$ the set $\left\{S_{0}, S_{1}, \ldots, S_{n}\right\}$ and will refer to it as the chain of numerical semigroups associated to $S$.

Proposition 2.2. Let $\mathcal{P}$ be a family of numerical semigroups with maximum $\Delta$. Then $\mathcal{P}$ is a pseudo-variety if and only if $\mathcal{P} \cup \mathcal{C}(\Delta)$ is a variety.

Proof. (Necessity.) Let us see that, if $S_{1}, S_{2} \in \mathcal{P} \cup \mathcal{C}(\Delta)$, then $S_{1} \cap S_{2} \in \mathcal{P} \cup \mathcal{C}(\Delta)$. This result is clear if $S_{1}, S_{2} \in \mathcal{P}$ or if $S_{1}, S_{2} \in \mathcal{C}(\Delta)$. Thereby, we can assume without lost of generality that $S_{1} \in \mathcal{P}$ and $S_{2} \in \mathcal{C}(\Delta)$. Then $S_{1} \subseteq \Delta \subseteq S_{2}$ and, consequently, $S_{1} \cap S_{2}=S_{1} \in \mathcal{P} \cup \mathcal{C}(\Delta)$.

Now, let us show that, if $S \in \mathcal{P} \cup \mathcal{C}(\Delta)$ and $S \varsubsetneqq \mathbb{N}$, then $S \cup\{\mathrm{F}(S)\} \in \mathcal{P} \cup \mathcal{C}(\Delta)$. On the one hand, by the definition of pseudo-variety, if $S \in \mathcal{P}$ and $S \neq \Delta$, then $S \cup\{\mathrm{F}(S)\} \in \mathcal{P}$. On the other hand, by the definition of chain associated to a numerical semigroup, if $S \in \mathcal{C}(\Delta)$, then $S \cup\{\mathrm{F}(S)\} \in \mathcal{C}(\Delta)$.

(Sufficiency.) Firstly, by hypothesis, we know that $\mathcal{P}$ has a maximum. In fact, $\Delta$ is the maximum.

Now, let us see that, if $S_{1}, S_{2} \in \mathcal{P}$, then $S_{1} \cap S_{2} \in \mathcal{P}$. To do this, we observe that $S_{1} \cap S_{2} \in \mathcal{P} \cup \mathcal{C}(\Delta)$ and, since $\Delta$ is the maximum of $\mathcal{P}$, that $S_{1}, S_{2} \subseteq \Delta$. Thus $S_{1} \cap S_{2} \subseteq \Delta$, and so we conclude that $S_{1} \cap S_{2} \in \mathcal{P}$. $\Delta$.

Finally, if $S \in \mathcal{P}$ and $S \neq \Delta$, then $S$ is strictly contained in $\Delta$ and $S \cup\{\mathrm{F}(S)\} \in \mathcal{P}$ because $S \cup\{\mathrm{F}(S)\} \subseteq$

The previous proposition allows us to give varieties from pseudo-varieties, as we can see in the following example.

Example 2.3. If $S$ is a numerical semigroup, then the multiplicity of $S$, denoted by $\mathrm{m}(S)$, is the least integer belonging to $S$. It is clear that the family

$$
\mathcal{P}(m)=\{S \mid S \text { is a numerical semigroup with } \mathrm{m}(S)=m\}
$$

is a pseudo-variety with $\max (\mathcal{P}(m))=\{0, m, \rightarrow\}$ (where the symbol " $\rightarrow$ " means that every integer greater than $m$ belongs to the set). Hence, applying Proposition 2.2, we have that $\mathcal{P}(m) \cup \mathcal{C}(\{0, m, \rightarrow\})=\mathcal{P}(m) \cup$ $\{\{0, m, \rightarrow\},\{0, m-1, \rightarrow\}, \ldots,\{0,1, \rightarrow\}\}$ is a variety.

Our next task in this section will be to prove Theorem 2.7. In order to do this we need to introduce some previous results. From now on, if $A$ is a set, then we denote by $\sharp A$ the cardinality of $A$.

Lemma 2.4. If $\mathcal{P}$ is a pseudo-variety and $S \in \mathcal{P}$, then $\max (\mathcal{P}) \in \mathcal{C}(S)$.

Proof. We will do the proof by induction over $\sharp(\max (\mathcal{P}) \backslash S)$. If $\sharp(\max (\mathcal{P}) \backslash S)=0$, then the result is trivially true. Now, let us suppose that $\sharp(\max (\mathcal{P}) \backslash S)>0$. Then $S \neq \max (\mathcal{P})$ and, thereby, $S \cup\{\mathrm{F}(S)\} \in \mathcal{P}$. Consequently, $S \cup\{\mathrm{F}(S)\} \subseteq \max (\mathcal{P})$ and $\sharp(\max (\mathcal{P}) \backslash(S \cup\{\mathrm{F}(S)\}))<\sharp(\max (\mathcal{P}) \backslash S)$. By applying the hypothesis of induction, we conclude that $\max (\mathcal{P}) \in \mathcal{C}(S \cup\{\mathrm{F}(S)\}) \subseteq \mathcal{C}(S)$.

The following result is straightforward to prove.

Lemma 2.5. If $S_{1}, \ldots, S_{r}$ are numerical semigroups, then

$$
\mathrm{F}\left(S_{1} \cap \ldots \cap S_{r}\right)=\max \left\{\mathrm{F}\left(S_{1}\right), \ldots, \mathrm{F}\left(S_{r}\right)\right\} .
$$

Lemma 2.6. Let $S_{1}, S_{2}, \Delta$ be numerical semigroups such that $\Delta \in \mathcal{C}\left(S_{1}\right)$ and $\Delta \in \mathcal{C}\left(S_{2}\right)$. Then $\Delta \in \mathcal{C}\left(S_{1} \cap S_{2}\right)$.

Proof. We will consider induction over $\sharp\left(\Delta \backslash\left(S_{1} \cap S_{2}\right)\right)$. If $\sharp\left(\Delta \backslash\left(S_{1} \cap S_{2}\right)\right)=0$, then $S_{1} \cap S_{2}=\Delta$ and, consequently, $\Delta \in \mathcal{C}\left(S_{1} \cap S_{2}\right)$. Now, let us suppose that $\sharp\left(\Delta \backslash\left(S_{1} \cap S_{2}\right)\right)>0$ and let $F=\mathrm{F}\left(S_{1} \cap S_{2}\right)$. By Lemma 2.5. for every $i \in\{1,2\}$, we deduce that $S_{i} \cup\{F\}=S_{i}$ or $S_{i} \cup\{F\}=S_{i} \cup\left\{\mathrm{F}\left(S_{i}\right)\right\}$. Therefore, $\Delta \in \mathcal{C}\left(S_{1} \cup\{F\}\right)$ and $\Delta \in \mathcal{C}\left(S_{2} \cup\{F\}\right)$. By applying the hypothesis of induction, we conclude that $\Delta \in$ $\mathcal{C}\left(\left(S_{1} \cup\{F\}\right) \cap\left(S_{2} \cup\{F\}\right)\right)=\mathcal{C}\left(\left(S_{1} \cap S_{2}\right) \cup\{F\}\right) \subseteq \mathcal{C}\left(S_{1} \cap S_{2}\right)$.

Now, we are in position to prove the result announced before.

Theorem 2.7. Let $\mathcal{V}$ be a variety and let $\Delta$ be a numerical semigroup such that $\Delta \in \mathcal{V}$. Then $\mathcal{D}(\mathcal{V}, \Delta)=$ $\{S \in \mathcal{V} \mid \Delta \in \mathcal{C}(S)\}$ is a pseudo-variety. Moreover, every pseudo-variety can be obtained in this way.

Proof. Let us check that $\mathcal{D}(\mathcal{V}, \Delta)$ satisfies the conditions of pseudo-variety.

- It is clear that $\Delta \in \mathcal{D}(\mathcal{V}, \Delta)$. Therefore, $\mathcal{D}(\mathcal{V}, \Delta)$ is not-empty. 
- If $S \in \mathcal{D}(\mathcal{V}, \Delta)$, then $S \subseteq \Delta$. Thus, $\Delta=\max (\mathcal{D}(\mathcal{V}, \Delta))$.

- If $S_{1}, S_{2} \in \mathcal{D}(\mathcal{V}, \Delta)$, then $S_{1} \cap S_{2} \in \mathcal{V}, \Delta \in \mathcal{C}\left(S_{1}\right)$, and $\Delta \in \mathcal{C}\left(S_{2}\right)$. From Lemma 2.6, we can assert that $S_{1} \cap S_{2} \in \mathcal{D}(\mathcal{V}, \Delta)$.

- If $S \in \mathcal{D}(\mathcal{V}, \Delta)$ and $S \neq \Delta$, then $S \cup\{\mathrm{F}(S)\} \in \mathcal{V}$ and $\Delta \in \mathcal{C}(S \cup\{\mathrm{F}(S)\})$ (because $S \cup\{\mathrm{F}(S)\} \subseteq \Delta)$. Therefore, $S \cup\{\mathrm{F}(S)\} \in \mathcal{D}(\mathcal{V}, \Delta)$.

Now, let $\mathcal{P}$ be a pseudo-variety with $\Delta=\max (\mathcal{P})$. From Proposition 2.2, we know that $\mathcal{V}=\mathcal{P} \cup \mathcal{C}(\Delta)$ is a variety. By applying Lemma 2.4, we conclude that $\mathcal{P}=\mathcal{D}(\mathcal{V}, \Delta)$.

The previous theorem allows us to construct pseudo-varieties from varieties such as we can see in the next example. From now on, by $\mathcal{L}$ we will denote the set of all numerical semigroups.

Example 2.8. It is clear that $\mathcal{L}$ is a variety. In addition, by Theorem 2.7 if $\Delta$ is a numerical semigroup, then $\mathcal{D}(\mathcal{L}, \Delta)=\{S \in \mathcal{L} \mid \Delta \in \mathcal{C}(S)\}$ is a pseudo-variety. For example, let us suppose that $\Delta=\{0, m, \rightarrow\}$, for some positive integer $m$. In this case, if $S$ is a numerical semigroup, then $\Delta \in \mathcal{C}(S)$ if and only if $S \subseteq \Delta$. Therefore, we get that $\mathcal{D}(\mathcal{L},\{0, m, \rightarrow\})=\{S \in \mathcal{L} \mid S \subseteq\{0, m, \rightarrow\}\}$ is a pseudo-variety.

\section{$3 \quad \mathcal{P}$-monoids}

From now on in this paper, by $\mathcal{P}$ we will denote a pseudo-variety. We will say that a submonoid $M$ of $(\mathbb{N},+)$ is a $\mathcal{P}$-monoid if it can be expressed as an intersection of elements of $\mathcal{P}$. The following result has an immediate proof.

Lemma 3.1. The intersection of $\mathcal{P}$-monoids is a $\mathcal{P}$-monoid.

If $A \subseteq \max (\mathcal{P})$, we will define the $\mathcal{P}$-monoid generated by $A$, denoted by $\mathcal{P}(A)$, as the intersection of all the $\mathcal{P}$-monoids containing $A$. As a consequence of Lemma 3.1, we have that $\mathcal{P}(A)$ is the smallest (with respect to the inclusion order) $\mathcal{P}$-monoid containing $A$. The next result is easy to prove.

Lemma 3.2. If $A \subseteq \max (\mathcal{P})$, then $\mathcal{P}(A)$ is the intersection of all elements of $\mathcal{P}$ containing $A$.

If $M=\mathcal{P}(A)$, then we will say that $A$ is a $\mathcal{P}$-system of generators of $M$. In addition, if $M \neq \mathcal{P}(B)$ for every $B \varsubsetneqq A$, then we will say that $A$ is a minimal $\mathcal{P}$-system of generators of $M$. The main goal of this section will be to prove that every $\mathcal{P}$-monoid admits a unique minimal $\mathcal{P}$-system of generators. In order to achieve this result, we need some previous lemmas. The first one is easily deduced from Lemma 3.2 .

Lemma 3.3. Let $A, B \subseteq \max (\mathcal{P})$ and let $M$ be a $\mathcal{P}$-monoid. Then we have that,

1. if $A \subseteq B$, then $\mathcal{P}(A) \subseteq \mathcal{P}(B)$;

2. $\mathcal{P}(A)=\mathcal{P}(\langle A\rangle)$;

3. $\mathcal{P}(M)=M$.

Let us see a result on existence and finiteness of the $\mathcal{P}$-system of generators.

Lemma 3.4. Every $\mathcal{P}$-monoid has a finite $\mathcal{P}$-system of generators.

Proof. Let $M$ be a $\mathcal{P}$-monoid. Then $M$ is a submonoid of $(\mathbb{N},+)$. Since every submonoid of $(\mathbb{N},+)$ is finitely generated, then there exists a finite set $A \subseteq M$ such that $M=\langle A\rangle$. By Lemma 3.3, we have that $M=\mathcal{P}(M)=\mathcal{P}(\langle A\rangle)=\mathcal{P}(A)$. Consequently, $A$ is a finite $\mathcal{P}$-system of generators of $M$.

Now, we show a characterization of the minimal $\mathcal{P}$-systems of generators, which is analogous to the characterization of minimal systems of generators for submonoids of $(\mathbb{N},+$ ) (see [3. Corollary 2.9]).

Lemma 3.5. If $A \subseteq \max (\mathcal{P})$ and $M=\mathcal{P}(A)$, then $A$ is a minimal $\mathcal{P}$-system of generators of $M$ if and only if $a \notin \mathcal{P}(A \backslash\{a\})$ for every $a \in A$. 
Proof. (Necessity.) If $a \in \mathcal{P}(A \backslash\{a\})$, then $A \subseteq \mathcal{P}(A \backslash\{a\})$. By Lemma 3.3, we get that $M=\mathcal{P}(A) \subseteq$ $\mathcal{P}(\mathcal{P}(A \backslash\{a\})) \subseteq \mathcal{P}(\mathcal{P}(A))=\mathcal{P}(M)=M$. Therefore, $M=\mathcal{P}(A \backslash\{a\})$, in contradiction with that $A$ is a minimal $\mathcal{P}$-system of generators of $M$.

(Sufficiency.) If $A$ is not a minimal $\mathcal{P}$-system of generators of $M$, then there exists $B \varsubsetneqq A$ such that $\mathcal{P}(B)=M$. Let $a \in A \backslash B$. By applying Lemma 3.3, we have that $a \in M=\mathcal{P}(B) \subseteq \mathcal{P}(A \backslash\{a\})$, in contradiction with the hypothesis.

Next we will see two technical lemmas, which are necessary for our purpose. The second one generalizes an evident property of submonoids of $\mathbb{N}$. More concretely, every element $x$ of a submonoid $M$ is expressible as a linear combination of the generators of $M$ that are smaller than or equal to $x$.

Lemma 3.6. If $S \in \mathcal{P}, x \in \max (\mathcal{P}) \backslash S$, and $y \geq x$, then $S \cup\{y, \rightarrow\} \in \mathcal{P}$.

Proof. We will do the proof by induction over $\sharp(\max (\mathcal{P}) \backslash S)$. First of all, let us observe that, since $\sharp(\max (\mathcal{P}) \backslash$ $S) \geq 1$, then $S \neq \max (\mathcal{P})$ and, consequently, $S \cup\{\mathrm{F}(S)\} \in \mathcal{P}$.

Let us consider $\sharp(\max (\mathcal{P}) \backslash S)=1$. Then $S \cup\{\mathrm{F}(S)\}=\max (\mathcal{P})$ and $x=\mathrm{F}(S)$. We have two possibilities.

1. If $y=x$, then $S \cup\{y, \rightarrow\}=S \cup\{\mathrm{F}(S), \rightarrow\}=S \cup\{\mathrm{F}(S)\} \in \mathcal{P}$.

2. If $y>x$, then $S \cup\{y, \rightarrow\}=S \in \mathcal{P}$.

Now, let $\sharp(\max (\mathcal{P}) \backslash S)=n+1$. Then $\sharp(\max (\mathcal{P}) \backslash(S \cup\{\mathrm{F}(S)\})=n$. On the one hand, if $x=\mathrm{F}(S)$, then the same reasoning as above allows us to assert that $S \cup\{y, \rightarrow\} \in \mathcal{P}$. On the other hand, if $x \neq \mathrm{F}(S)$, then $x \in \max (\mathcal{P}) \backslash(S \cup\{\mathrm{F}(S)\})$ and, by applying the hypothesis of induction, we get that $S \cup\{\mathrm{F}(S)\} \cup\{y, \rightarrow\} \in \mathcal{P}$. Being that $S \cup\{y, \rightarrow\}$ is equal to

- $S \cup\{\mathrm{F}(S)\}$ if $y=\mathrm{F}(S)$,

- $S$ if $y>\mathrm{F}(S)$,

- and $S \cup\{\mathrm{F}(S)\} \cup\{y, \rightarrow\}$ if $y<\mathrm{F}(S)$,

then we can conclude that $S \cup\{y, \rightarrow\} \in \mathcal{P}$.

Lemma 3.7. If $A \subseteq \max (\mathcal{P})$ and $x \in \mathcal{P}(A)$, then $x \in \mathcal{P}(\{a \in A \mid a \leq x\})$.

Proof. Let us suppose that $x \notin \mathcal{P}(\{a \in A \mid a \leq x\})$. By Lemma 3.2, we have that there exists $S \in \mathcal{P}$ such that $\{a \in A \mid a \leq x\} \subseteq S$ and $x \notin S$. Since $x \in \mathcal{P}(A)$, then $x \in \max (\mathcal{P})$ and, thereby, $x \in \max (\mathcal{P}) \backslash S$. Thus, by applying Lemma 3.6 we have that $S \cup\{x+1, \rightarrow\} \in \mathcal{P}$. However, since obviously $A \subseteq S \cup\{x+1, \rightarrow\}$ and $x \notin S \cup\{x+1, \rightarrow\}$, by applying once more Lemma 3.2. we get that $x \notin \mathcal{P}(A)$, in contradiction with the hypothesis.

We are now ready to present the result of uniqueness.

Theorem 3.8. Let $M$ be a $\mathcal{P}$-monoid. If $A, B$ are two minimal $\mathcal{P}$-systems of generators of $M$, then $A=B$.

Proof. Let us suppose that $A=\left\{a_{1}<a_{2}<\cdots\right\}$ and $B=\left\{b_{1}<b_{2}<\cdots\right\}$. If $A \neq B$, then there exists $i=\min \left\{k \mid a_{k} \neq b_{k}\right\}$. Let us assume, without loss of generality, that $a_{i}<b_{i}$. Since $a_{i} \in M=\mathcal{P}(A)=\mathcal{P}(B)$, by Lemma 3.7, we have that $a_{i} \in \mathcal{P}\left(\left\{b_{1}, \ldots, b_{i-1}\right\}\right)$. Being that, by the minimality of $i,\left\{b_{1}, \ldots, b_{i-1}\right\}=$ $\left\{a_{1}, \ldots, a_{i-1}\right\}$, then $a_{i} \in \mathcal{P}\left(\left\{a_{1}, \ldots, a_{i-1}\right\}\right)$. Therefore, we conclude that $a_{i} \in \mathcal{P}\left(A \backslash\left\{a_{i}\right\}\right)$, which is a contradiction with Lemma 3.5 .

As an immediate consequence of Theorem 3.8 we establish the following result.

Corollary 3.9. Every $\mathcal{P}$-monoid has a unique minimal $\mathcal{P}$-system of generators. Additionally, such a $\mathcal{P}$-system is finite.

If $M$ is a $\mathcal{P}$-monoid, then the cardinality of the minimal $\mathcal{P}$-system of generators of $M$ will be called the $\mathcal{P}$-range of $M$.

We finish this section with two illustrative examples of its content.

Example 3.10. Let $\mathcal{P}=\{S \in \mathcal{L} \mid \mathrm{m}(S)=4\}$. By Example 2.3 we know that $\mathcal{P}$ is a pseudo-variety. We are going to compute the minimal $\mathcal{P}$-system of generators of the submonoid of $(\mathbb{N},+)$ generated by $\{4,6,7\}$, that is, of the submonoid $M=\langle 4,6,7\rangle=\{0,4,6,7,8,10, \rightarrow\}$. Observe that $M \in \mathcal{P}$ and, therefore, $M$ is a $\mathcal{P}$-monoid. 
- First of all, let us see that $\mathcal{P}(\{6,7\})=M$. Indeed, if $S \in \mathcal{P}$ and $\{6,7\} \subseteq S$, then $\{4,6,7\} \subseteq S$. Thereby, $\langle 4,6,7\rangle \subseteq S$. By Lemma 3.2 , we have that $\mathcal{P}(\{6,7\})=M$.

- Now, let us see that $\mathcal{P}(\{6\}) \neq M$. Since $\langle 4,6,9\rangle \in \mathcal{P}$ and $\{6\} \subseteq\langle 4,6,9\rangle$, by Lemma 3.2, we have that $\mathcal{P}(\{6\}) \subseteq\langle 4,6,9\rangle$. However, $7 \notin\langle 4,6,9\rangle$. By applying again Lemma 3.2, we can conclude that $\mathcal{P}(\{6\}) \neq M$.

- Finally, in order to see that $\mathcal{P}(\{7\}) \neq M$, it is enough to observe that $\langle 4,7\rangle \in \mathcal{P},\{7\} \subseteq\langle 4,7\rangle$, and $6 \notin\langle 4,7\rangle$.

From the previous comments, we have that $\{6,7\}$ is the minimal $\mathcal{P}$-system of generators of $M$ and, moreover, the $\mathcal{P}$-range of $M$ is equal to 2 .

Example 3.11. It is interesting to observe that a (minimal) $\mathcal{P}$-system of generators can be empty. In effect, let $\mathcal{P}$ be the same pseudo-variety as in Example 3.10. If $S_{k}=\langle 4,4 k+5\rangle$ for all $k \in \mathbb{N}$, then it is clear that $M=\bigcap_{k \in \mathbb{N}} S_{k}=\langle 4\rangle$ is a $\mathcal{P}$-monoid. Since $\mathcal{P}(\emptyset)=M$, we conclude that the minimal $\mathcal{P}$-system of generators of $M=\langle 4\rangle$ is empty, that is, the $\mathcal{P}$-range of $M=\langle 4\rangle$ is equal to 0 .

\section{The tree associated to a pseudo-variety}

A graph $G$ is a pair $(V, E)$, where $V$ is a non-empty set and $E$ is a subset of $\{(v, w) \in V \times V \mid v \neq w\}$. The elements of $V$ are called vertices of $G$ and the elements of $E$ are called edges of $G$.

A path (of length $n$ ) connecting the vertices $x$ and $y$ of $G$ is a sequence of different edges of the form $\left(v_{0}, v_{1}\right),\left(v_{1}, v_{2}\right), \ldots,\left(v_{n-1}, v_{n}\right)$ such that $v_{0}=x$ and $v_{n}=y$.

A graph $G$ is a tree if there exist a vertex $r$ (known as the root of $G$ ) such that, for every other vertex $x$ of $G$, there exists a unique path connecting $x$ and $r$. If there exists a path connecting the vertices $x$ and $y$, then we say that $x$ is a descendant of $y$. In particular, if $(x, y)$ is an edge of the tree, then we say that $x$ is a son of $y$.

For a pseudo-variety $\mathcal{P}$, we define the graph $\mathrm{G}(\mathcal{P})$ in the following way,

- the set of vertices of $\mathrm{G}(\mathcal{P})$ is $\mathcal{P}$;

- $\left(S, S^{\prime}\right) \in \mathcal{P} \times \mathcal{P}$ is an edge of $\mathrm{G}(\mathcal{P})$ if and only if $S^{\prime}=S \cup\{\mathrm{F}(S)\}$.

If $S \in \mathcal{P}$, then we can define recursively the sequence,

- $S_{0}=S$,

- if $S_{i} \neq \max (\mathcal{P})$, then $S_{i+1}=S_{i} \cup\left\{\mathrm{F}\left(S_{i}\right)\right\}$.

As a consequence of Lemma 2.4 we have a chain of elements in $\mathcal{P}$. Namely, $S=S_{0} \varsubsetneqq S_{1} \varsubsetneqq \cdots \varsubsetneqq S_{n}=\max (\mathcal{P})$. As an application of the above comment, we have the next result.

Lemma 4.1. $\mathrm{G}(\mathcal{P})$ is a tree with root $\max (\mathcal{P})$.

Observe that we can construct recursively $\mathrm{G}(\mathcal{P})$ from $\max (\mathcal{P})$. Indeed, it is sufficient to compute the sons of each vertex $S$. Thus, if $T$ is a son of $S$ in $\mathrm{G}(\mathcal{P})$, then $S=T \cup\{\mathrm{F}(T)\}$ and, thereby, $T=S \backslash\{\mathrm{F}(T)\}$. Consequently, if $T$ is a son of $S$ in $\mathrm{G}(\mathcal{P})$, then there exists an integer $x>\mathrm{F}(S)$ such that $T=S \backslash\{x\}$.

The following lemma is the analogous of a well known result for numerical semigroups.

Lemma 4.2. Let $M$ be a $\mathcal{P}$-monoid and let $x \in M$. Then $M \backslash\{x\}$ is a $\mathcal{P}$-monoid if and only if $x$ belongs to the minimal $\mathcal{P}$-system of generators of $M$.

Proof. Let $A$ be the minimal $\mathcal{P}$-system of generators of $M$. If $x \notin A$, then $A \subseteq M \backslash\{x\}$. Since $M \backslash\{x\}$ is a $\mathcal{P}$-monoid containing $A$, we have that $M=\mathcal{P}(A) \subseteq M \backslash\{x\}$, which is a contradiction.

Conversely, by Theorem 3.8 we have that, if $x \in A$, then $\mathcal{P}(M \backslash\{x\}) \neq \mathcal{P}(A)=M$. Thereby, $M \backslash\{x\} \subseteq$ $\mathcal{P}(M \backslash\{x\}) \varsubsetneqq M$ and, therefore, $\mathcal{P}(M \backslash\{x\})=M \backslash\{x\}$. Consequently, $M \backslash\{x\}$ is a $\mathcal{P}$-monoid.

As an immediate consequence of the results and comments of this section, we have the next result.

Theorem 4.3. The graph $\mathrm{G}(\mathcal{P})$ is a tree with root equal to $\max (\mathcal{P})$. Moreover, the sons of a vertex $S \in \mathcal{P}$ are $S \backslash\left\{x_{1}\right\}, \ldots, S \backslash\left\{x_{r}\right\}$, where $x_{1}, \ldots, x_{r}$ are the elements of the minimal $\mathcal{P}$-system of generators of $S$ that are greater than $\mathrm{F}(S)$. 
We illustrate the previous results with an example.

Example 4.4. Let $\Delta$ be a numerical semigroup different from $\mathbb{N}$. Let us denote by

$$
\mathcal{L}(\Delta,\langle 2,3\rangle)=\{S \in \mathcal{L} \mid \Delta \subseteq S \subseteq\langle 2,3\rangle\}
$$

It is clear that $\mathcal{L}(\Delta,\langle 2,3\rangle)$ is a pseudo-variety with $\max (\mathcal{L}(\Delta,\langle 2,3\rangle))=\langle 2,3\rangle$. Moreover, by Lemma 4.2 , we easily deduce that, if $S \in \mathcal{L}(\Delta,\langle 2,3\rangle)$, then the minimal $\mathcal{L}(\Delta,\langle 2,3\rangle)$-generators of $S$ are precisely the minimal generators of $S$ that do not belong to $\Delta$. Thus, applying Theorem 4.3 , we have that $\mathrm{G}(\mathcal{L}(\langle 4,5,6\rangle,\langle 2,3\rangle))$ is given by the following tree.

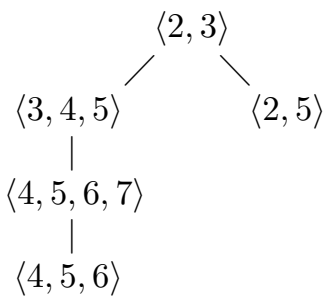

As an immediate consequence of Theorem 4.3. we will give an algorithm which allows us to compute, from a given pseudo-variety $\mathcal{P}$, the set of all elements of $\mathcal{P}$ with a certain fixed genus. We will denote by $\mathcal{P}(g)$ the set of all elements of $\mathcal{P}$ that have genus equal to $g$. It is clear that, if $g<\mathrm{g}(\max (\mathcal{P}))$, then $\mathcal{P}(g)=\emptyset$.

Algorithm 4.5. Let $\mathcal{P}$ be a pseudo-variety.

INPUT: A positive integer $g$.

OUTPUT: $\mathcal{P}(g)$.

(1) If $g<\mathrm{g}(\max (\mathcal{P}))$, then return $\emptyset$.

(2) Set $A=\{\max (\mathcal{P})\}$ and $i=g(\max (\mathcal{P}))$.

(3) If $i=g$, then return $A$.

(4) For each $S \in A$, compute the set $B_{S}$ formed by all elements of the minimal $\mathcal{P}$-system of generators of $S$ that are greater than $\mathrm{F}(S)$.

(5) If $\bigcup_{S \in A} B_{S}=\emptyset$, then return $\emptyset$.

(6) Set $A=\bigcup_{S \in A}\left\{S \backslash\{x\} \mid x \in B_{S}\right\}, i=i+1$, and go to (3).

We illustrate the operation of this algorithm with an example.

Example 4.6. Let $\mathcal{P}=\mathcal{L}(\langle 4,5,6\rangle,\langle 2,3\rangle)=\{S \in \mathcal{L} \mid\langle 4,5,6\rangle \subseteq S \subseteq\langle 2,3\rangle\}$. By Example 4.4, we know that $\mathcal{P}$ is a pseudo-variety. Moreover, we also know that, if $S \in \mathcal{P}$, then the minimal $\mathcal{P}$-generators of $S$ are the minimal generators of $S$ that do not belong to $\langle 4,5,6\rangle$. We are going to compute $\mathcal{P}(3)$, that is, all elements of $\mathcal{P}$ with genus equal to 3 . Observe that $\mathrm{g}(\max (\mathcal{P}))=\mathrm{g}(\langle 2,3\rangle)=1$.

- $A=\{\langle 2,3\rangle\}, i=1$.

- $B_{\langle 2,3\rangle}=\{2,3\}$.

- $A=\{\langle 3,4,5\rangle,\langle 2,5\rangle\}, \mathrm{i}=2$.

- $B_{\langle 3,4,5\rangle}=\{3\}, B_{\langle 2,5\rangle}=\emptyset$.

- $A=\{\langle 4,5,6,7\rangle\}, i=3$.

- $\mathcal{P}(3)=\{\langle 4,5,6,7\rangle\}$.

Let us remember that, by Theorem 2.7, if $\mathcal{V}$ is a variety and $\Delta \in \mathcal{V}$, then $\mathcal{D}(\mathcal{V}, \Delta)=\{S \in \mathcal{V} \mid \Delta \in \mathcal{C}(S)\}$ is a pseudo-variety and, moreover, every pseudo-variety can be obtained in this way. On the other hand, let us observe that $\mathcal{D}(\mathcal{V}, \Delta)$ is formed by the descendants of $\Delta$ in the tree $\mathrm{G}(\mathcal{V})$. Therefore, we can think in a pseudo-variety as a subtree obtained from the tree of a variety when we take a vertex and all its descendants. 
Example 4.7. Being $\mathcal{L}$ the variety formed by all numerical semigroups, it is clear that the concept of minimal $\mathcal{L}$-system of generators coincide with the usual concept of minimal system of generators. Thus, it is not difficult to see that the descendants of the numerical semigroup $\langle 5,6,8,9\rangle$, in the tree $\mathrm{G}(\mathcal{L})$, are given by the next subtree.

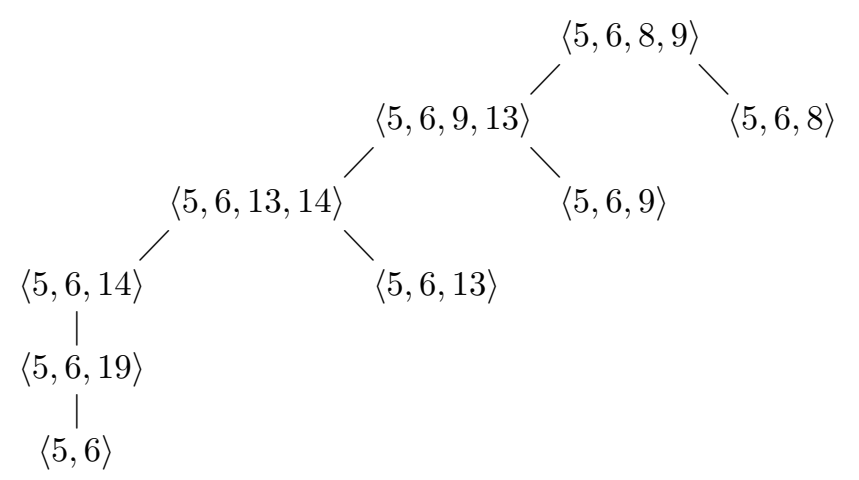

Therefore, $\mathcal{P}=\{\langle 5,6,8,9\rangle,\langle 5,6,9,13\rangle,\langle 5,6,8\rangle,\langle 5,6,13,14\rangle,\langle 5,6,9\rangle,\langle 5,6,14\rangle,\langle 5,6,13\rangle,\langle 5,6,19\rangle,\langle 5,6\rangle\}$ is a pseudo-variety.

\section{The pseudo-variety generated by a family of numerical semi- groups}

In general, the intersection of pseudo-varieties is not a pseudo-variety. Indeed, if $S$ and $T$ are different numerical semigroups, then $\mathcal{P}_{1}=\{S\}$ and $\mathcal{P}_{2}=\{T\}$ are pseudo-varieties, but not $\mathcal{P}_{1} \cap \mathcal{P}_{2}=\{\emptyset\}$.

The proofs of the next two lemmas are straightforward.

Lemma 5.1. Let $\left\{\mathcal{P}_{i}\right\}_{i \in I}$ be a family of pseudo-varieties such that $\max \left(\mathcal{P}_{i}\right)=\Delta$ for every $i \in I$. Then $\bigcap_{i \in I} \mathcal{P}_{i}$ is a pseudo-variety and $\max \left(\bigcap_{i \in I} \mathcal{P}_{i}\right)=\Delta$.

Lemma 5.2. Let $S, S^{\prime}$ be numerical semigroups. Then $S^{\prime} \in \mathcal{C}(S)$ if and only if $S^{\prime}=S \cup\{x, \rightarrow\}$ for some positive integer $x$.

The following two lemmas will allow us to determine the maximum element for the pseudo-varieties we consider in this section.

Lemma 5.3. Let $\mathcal{F}$ be a non-empty family of numerical semigroups. Then $\Delta \in \bigcap_{S \in \mathcal{F}} \mathcal{C}(S)$ if and only if there exists a positive integer $x$ such that $\Delta=S \cup\{x, \rightarrow\}$ for every $S \in \mathcal{F}$.

Proof. (Necessity). Applying Lemma 5.2, we have that, if $\Delta \in \bigcap_{S \in \mathcal{F}} \mathcal{C}(S)$, then for each $S \in \mathcal{F}$ there exists a positive integer $x_{S}$ such that $\Delta=S \cup\left\{x_{S}, \rightarrow\right\}$. Now, if $x=\min \left\{x_{S} \mid S \in \mathcal{F}\right\}$, then $\{x, \rightarrow\} \subseteq \Delta$ and, therefore, $S \cup\{x, \rightarrow\}=\Delta$ for every $S \in \mathcal{F}$.

(Sufficiency). It is consequence of Lemma 5.2 .

Lemma 5.4. Let $\mathcal{F}$ be a non-empty family of numerical semigroups. Then there exists the minimum (with respect to the inclusion order) of the set $\bigcap_{S \in \mathcal{F}} \mathcal{C}(S)$. Moreover, if $\Theta$ is such a minimum, then $\bigcap_{S \in \mathcal{F}} \mathcal{C}(S)=$ $\mathcal{C}(\Theta)$.

Proof. If $\sharp \mathcal{F}=1$, then $\mathcal{F}=\{S\}$ for some numerical semigroup $S$ and, in addition, $S=\min (\mathcal{C}(S))$. Therefore, we can suppose that $\sharp \mathcal{F} \geq 2$. If this is the case, then $\bigcap_{S \in \mathcal{F}} S \subsetneq \bigcup_{S \in \mathcal{F}} S$ and, thereby, there exists $a=$ $\min \left(\bigcup_{S \in \mathcal{F}} S \backslash \bigcap_{S \in \mathcal{F}} S\right)$.

Let $\Delta \in \bigcap_{S \in \mathcal{F}} \mathcal{C}(S)$. Then, by Lemma 5.3 . we know that there exists a positive integer $x$ such that $S \cup\{x, \rightarrow\}=\Delta$ for every $S \in \mathcal{F}$.

Let us see that $x \leq a$. Indeed, by the choice of $a$, we know that there exist $S_{1}, S_{2} \in \mathcal{F}$ such that $a \in S_{1}$ and $a \notin S_{2}$. Consequently, if $x>a$, then $S_{1} \cup\{x, \rightarrow\} \neq S_{2} \cup\{x, \rightarrow\}$, that is, a contradiction.

Again by the choice of $a$, we can observe that, if $x \leq a$, then $T \cup\{x, \rightarrow\}=\left(\bigcap_{S \in \mathcal{F}} S\right) \cup\{x, \rightarrow\}$ for every $T \in \mathcal{F}$.

From here, it is not difficult to deduce that $\Theta=\left(\bigcap_{S \in \mathcal{F}} S\right) \cup\{a, \rightarrow\}$ is the minimum of $\bigcap_{S \in \mathcal{F}} \mathcal{C}(S)$ and that $\mathcal{C}(\Theta)=\bigcap_{S \in \mathcal{F}} \mathcal{C}(S)$. 
Let $\mathcal{F}$ be a non-empty family of numerical semigroups. Then we will denote by $\Theta(\mathcal{F})$ the numerical semigroup $\min \left(\bigcap_{S \in \mathcal{F}} \mathcal{C}(S)\right)$. Moreover, by $\mathfrak{p}(\mathcal{F})$ we will denote the intersection of all pseudo-varieties that contain $\mathcal{F}$ and whose maximum is $\Theta(\mathcal{F})$. Observe that there always exists, at least, one pseudo-variety with these characteristics. In effect, by Theorem 2.7, we know that $\mathcal{D}(\mathcal{L}, \Theta(\mathcal{F}))$ is a pseudo-variety that contains $\mathcal{F}$ and with maximum equal to $\Theta(\mathcal{F})$.

The proof of the following result is the same as that of Theorem 2.7.

Lemma 5.5. If $\mathcal{P}$ is a pseudo-variety and $\Delta \in \mathcal{P}$, then $\mathcal{D}(\mathcal{P}, \Delta)=\{S \in \mathcal{P} \mid \Delta \in \mathcal{C}(S)\}$ is a pseudo-variety with maximum $\Delta$.

The next lemma has an immediate proof.

Lemma 5.6. Let $\mathcal{P}$ be a pseudo-variety, $S \in \mathcal{P}$, and let $x$ be a positive integer such that $S \cup\{x, \rightarrow\} \in \mathcal{P}$. If $y$ is a positive integer greater than or equal to $x$, then $S \cup\{y, \rightarrow\} \in \mathcal{P}$.

Lemma 5.7. Let $\mathcal{F}$ be a non-empty family of numerical semigroups and let $\mathcal{P}$ be a pseudo-variety such that $\mathcal{F} \subseteq \mathcal{P}$. Then $\Theta(\mathcal{F}) \in \mathcal{P}$.

Proof. By Lemma 2.4 , we know that $\max (\mathcal{P}) \in \bigcap_{S \in \mathcal{F}} \mathcal{C}(S)$ and, applying Lemma 5.4 , we have that $\max (\mathcal{P}) \in$ $\mathcal{C}(\Theta(\mathcal{F}))$. Now, by Lemma 5.2, if $S \in \mathcal{F}$, then there exists a positive integer $x$ such that $S \cup\{x, \rightarrow\}=\max (\mathcal{P})$ and there exists a positive integer $y$ such that $S \cup\{y, \rightarrow\}=\Theta(\mathcal{F})$. Since $\max (\mathcal{P}) \in \mathcal{C}(\Theta(\mathcal{F}))$, we have that $x \leq y$. The proof finishes by applying Lema 5.6.

Now, we are ready to show the main result of this section.

Theorem 5.8. If $\mathcal{F}$ is a non-empty family of numerical semigroups, then $\mathfrak{p}(\mathcal{F})$ is the smallest (with respect to the inclusion order) pseudo-variety containing $\mathcal{F}$.

Proof. By Lemma 5.1, we know that $\mathfrak{p}(\mathcal{F})$ is a pseudo-variety and that $\max (\mathfrak{p}(\mathcal{F}))=\Theta(\mathcal{F})$. Even more, we can assert that $\mathfrak{p}(\mathcal{F})$ is the smallest pseudo-variety containing $\mathcal{F}$ and such that $\Theta(\mathcal{F})$ is its maximum.

Let $\mathcal{P}^{\prime}$ be another pseudo-variety containing $\mathcal{F}$. Let us see that $\mathfrak{p}(\mathcal{F}) \subseteq \mathcal{P}^{\prime}$. In fact, since $\mathcal{F} \subseteq \mathcal{P}^{\prime}$, by Lemma 5.7 we know that $\Theta(\mathcal{F}) \in \mathcal{P}^{\prime}$. Thus, by Lemma 5.5 we have that $\mathcal{D}\left(\mathcal{P}^{\prime}, \Theta(\mathcal{F})\right)$ is a pseudo-variety with maximum $\Theta(\mathcal{F})$. Thereby, we conclude that $\mathfrak{p}(\mathcal{F}) \subseteq \mathcal{D}\left(\mathcal{P}^{\prime}, \Theta(\mathcal{F})\right) \subseteq \mathcal{P}^{\prime}$.

We will call $\mathfrak{p}(\mathcal{F})$ the pseudo-variety generated by $\mathcal{F}$. On the other hand, we will say that a pseudo-variety $\mathcal{P}$ is finitely generated if there exists a finite family of numerical semigroups, $\mathcal{F}$, such that $\mathcal{P}=\mathfrak{p}(\mathcal{F})$.

Let $\mathcal{F}$ be a non-empty family of numerical semigroups. If $S \in \mathcal{F}$, then we define recursively the following sequence of numerical semigroups (observe the analogy with the chain of numerical semigroups associated to $S)$

- $S_{0}=S$,

- if $S_{i} \neq \Theta(\mathcal{F})$, then $S_{i+1}=S_{i} \cup\left\{\mathrm{F}\left(S_{i}\right)\right\}$.

In this way, we obtain a chain of elements of $\mathfrak{p}(\mathcal{F})$, namely, $S=S_{0} \varsubsetneqq S_{1} \varsubsetneqq \cdots \varsubsetneqq S_{n}=\Theta(\mathcal{F})$. We will denote by $\mathcal{C}^{\mathcal{F}}(S)=\left\{S_{0}, S_{1}, \ldots, S_{n}\right\}$. If $A \subseteq \mathcal{F}$, then we will define $\mathcal{C}^{\mathcal{F}}(A)=\bigcup_{S \in A} \mathcal{C}^{\mathcal{F}}(S)$.

Proposition 5.9. Let $\mathcal{F}$ be a non-empty family of numerical semigroups. Then $\mathfrak{p}(\mathcal{F})$ is the set formed by all finite intersections of elements of $\mathcal{C}^{\mathcal{F}}(\mathcal{F})$.

Proof. Let $X=\left\{S_{1} \cap \cdots \cap S_{n} \mid n \in \mathbb{N} \backslash\{0\}\right.$ and $\left.S_{1}, \ldots, S_{n} \in \mathcal{C}^{\mathcal{F}}(\mathcal{F})\right\}$. From the definition of $\mathfrak{p}(\mathcal{F})$, it is clear that $X \subseteq \mathfrak{p}(\mathcal{F})$.

Let us now see that $\mathfrak{p}(\mathcal{F}) \subseteq X$. Having in mind that, by Theorem $5.8, \mathfrak{p}(\mathcal{F})$ is the smallest pseudo-variety containing $\mathcal{F}$, in order to get that inclusion, it will be enough to show that $X$ is a pseudo-variety. (Obviously, $\mathcal{F} \subseteq X)$. First of all, it is clear that $\Theta(\mathcal{F})$ is the maximum of $X$ and that, if $S_{1}, S_{2} \in X$, then $S_{1} \cap S_{2} \in X$. Now, let $S \in X$ such that $S \neq \Theta(\mathcal{F})$. Then there exist $S_{1}, \ldots, S_{n} \in \mathcal{C}^{\mathcal{F}}(\mathcal{F})$ such that $S=S_{1} \cap \cdots \cap S_{n}$. By Lemma 2.5. we have that $S_{i} \cup\{\mathrm{F}(S)\}=S_{i}$ or $S_{i} \cup\{\mathrm{F}(S)\}=S_{i} \cup\left\{\mathrm{F}\left(S_{i}\right)\right\}$. Consequently, we deduce that $S \cup\{\mathrm{F}(S)\}=\left(S_{1} \cup\{\mathrm{F}(S)\}\right) \cap \cdots \cap\left(S_{n} \cup\{\mathrm{F}(S)\}\right)$ and that $S_{i} \cup\{\mathrm{F}(S)\} \in \mathcal{C}^{\mathcal{F}}(\mathcal{F})$. Therefore, $S \cup\{\mathrm{F}(S)\} \in X$.

As an immediate consequence of the previous proposition we have the following result.

Corollary 5.10. A pseudo-variety is finitely generated if and only if it is finite. 
We finish this section showing a method which allows us to compute, beginning with $\mathcal{F}$, the minimal $\mathfrak{p}(\mathcal{F})$-system of generators of a $\mathfrak{p}(\mathcal{F})$-monoid. In order to do that, we need an immediate consequence of Proposition 5.9 and Lemma 3.2 .

Lemma 5.11. Let $\mathcal{F}$ be a non-empty family of numerical semigroups, $\mathcal{P}=\mathfrak{p}(\mathcal{F})$, and $A \subseteq \max (\mathcal{P})=\Theta(\mathcal{F})$. For each $S \in \mathcal{F}$ we define

$$
\alpha(S)=\left\{\begin{array}{l}
S, \text { if } A \subseteq S, \\
S \cup\left\{x_{S}, \rightarrow\right\}, \text { if } A \nsubseteq S,
\end{array}\right.
$$

where $x_{S}=\min \{a \in A \mid a \notin S\}$. Then $\mathcal{P}(A)=\bigcap_{S \in \mathcal{F}} \alpha(S)$.

Proposition 5.12. Let $\mathcal{F}$ be a non-empty family of numerical semigroups, $\mathcal{P}=\mathfrak{p}(\mathcal{F})$, and $A \subseteq \max (\mathcal{P})=$ $\Theta(\mathcal{F})$. For each $S \in \mathcal{F}$ such that $A \nsubseteq S$, let $x_{S}=\min \{a \in A \mid a \notin S\}$. Then $B=\left\{x_{S} \mid S \in \mathcal{F}\right.$ and $\left.A \nsubseteq S\right\}$ is the minimal $\mathcal{P}$-system of generators of $\mathcal{P}(A)$.

Proof. Observe that, if $S \in \mathcal{F}$, then $A \subseteq S$ if and only if $B \subseteq S$. Moreover, if $A \nsubseteq S$, then $\min \{a \in A \mid a \notin$ $S\}=\min \{b \in B \mid b \notin S\}$ and, by applying Lemma 5.11, $\mathcal{P}(A)=\mathcal{P}(B)$.

Now, in order to finish the proof (that is, $B$ is the minimal $\mathcal{P}$-system of generators of $\mathcal{P}(A)$ ), it will be enough to show that, if $C \subsetneq B$, then $\mathcal{P}(C) \neq \mathcal{P}(A)$. So, let $C \subsetneq B$. Then there exists $S \in \mathcal{F}$ such that $x_{S} \notin C$ and, thereby, $C \subseteq S$ or $\min \{c \in C \mid c \notin S\}>x_{S}$. From Lemma 5.11, we get that $x_{S} \notin \mathcal{P}(C)$. Finally, since $x_{S} \in B \subseteq A$, then $A \nsubseteq \mathcal{P}(C)$ and, therefore, $\mathcal{P}(C) \neq \mathcal{P}(A)$.

From the previous proposition, we easily deduce the next result.

Corollary 5.13. Let $\mathcal{F}$ be a non-empty family of numerical semigroups. Then every $\mathfrak{p}(\mathcal{F})$-monoid has $\mathfrak{p}(\mathcal{F})$ range less than or equal to $\sharp \mathcal{F}$.

We finish this section with an example which illustrates its content.

Example 5.14. Let $\mathcal{F}=\{\langle 5,6\rangle,\langle 5,6,8\rangle\}$. Let us compute the tree $\mathrm{G}(\mathfrak{p}(\mathcal{F}))$. To do this, if we compute $\mathcal{C}(\langle 5,6\rangle)$ and $\mathcal{C}(\langle 5,6,8\rangle)$, then it is easy to see that $\Theta(\mathcal{F})=\langle 5,6,8,9\rangle$. By using Theorem 4.3, we will show that the tree is as follows.

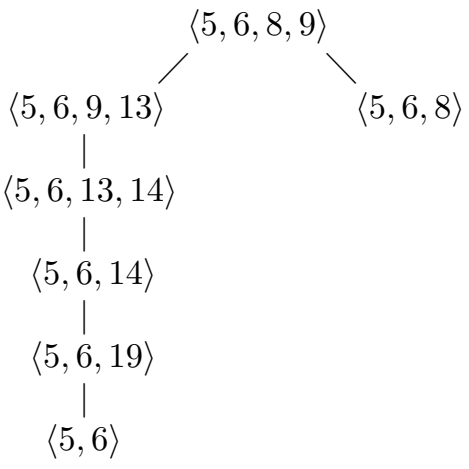

In order to compute the minimal $\mathfrak{p}(\mathcal{F})$-system of generators of $\langle 5,6,8,9\rangle$, we apply Proposition 5.12 with $A=\{5,6,8,9\}$. Since $x_{\langle 5,6\rangle}=8$ and $x_{\langle 5,6,8\rangle}=9$, then $\{8,9\}$ is the searched minimal $\mathfrak{p}(\mathcal{F})$-system. Now, since $\mathrm{F}(\langle 5,6,8,9\rangle)=7$, by Theorem $4.3\langle 5,6,8,9\rangle$ has two sons, $\langle 5,6,8,9\rangle \backslash\{8\}=\langle 5,6,9,13\rangle$ and $\langle 5,6,8,9\rangle \backslash\{9\}=\langle 5,6,8\rangle$.

Observe that $\langle 5,6,8\rangle$ has not got any son because the minimal $\mathfrak{p}(\mathcal{F})$-system of generators of $\langle 5,6,8\rangle$ is a subset of $\{5,6,8\}$ and $\mathrm{F}(\langle 5,6,8\rangle)=9$.

If we apply Proposition 5.12 with $A=\{5,6,9,13\}$, then we have that $\{9\}$ is the minimal $\mathfrak{p}(\mathcal{F})$-system of generators of $\langle 5,6,9,13\rangle$. Since $\mathrm{F}(\langle 5,6,9,13\rangle)=8$, then $\langle 5,6,9,13\rangle$ has a unique son, $\langle 5,6,9,13\rangle \backslash\{9\}=$ $\langle 5,6,13,14\rangle$.

By applying iteratively the reasoning of the previous paragraph, we get that

- $\{13\}$ is the minimal $\mathfrak{p}(\mathcal{F})$-system of generators of $\langle 5,6,13,14\rangle$ and $\langle 5,6,14\rangle$ is its unique son;

- $\{14\}$ is the minimal $\mathfrak{p}(\mathcal{F})$-system of generators of $\langle 5,6,14\rangle$ and $\langle 5,6,19\rangle$ is its unique son;

- $\{19\}$ is the minimal $\mathfrak{p}(\mathcal{F})$-system of generators of $\langle 5,6,19\rangle$ and $\langle 5,6\rangle$ is its unique son.

Finally, $\langle 5,6\rangle$ has not got any son because, in its minimal $\mathfrak{p}(\mathcal{F})$-system of generators, there is not any element greater than its Frobenius number. 


\section{References}

[1] M. Bras-Amorós, P. A. García-Sánchez, and A. Vico-Oton: Nonhomogeneous patterns on numerical semigroups. To appear in Internat. J. Algebra Comput. DOI:10.1142/S0218196713500306

[2] J. C. Rosales: Families of numerical semigroups closed under finite intersections and for the Frobenius number. Houston J. Math. 34, 339-348 (2008)

[3] J. C. Rosales and P. A. García-Sánchez: Numerical semigroups. Developments in Mathematics, vol. 20, Springer, New York (2009)

[4] J. C. Rosales, P. A. García-Sánchez, J. I. García-García and M. B. Branco: Numerical semigroups with maximal embedding dimension. Int. J. Commutative Rings 2, 47-53 (2003) 